\title{
Complex Fuzzy Groups Based On Rosenfeld's Approach
}

\author{
EMAN A. ABUHIJLEH \\ Department of Basic Sciences, Al-Zarqa University College, \\ Al-Balqa Applied University \\ JORDAN \\ MOURAD MASSA'DEH \\ Department of Applied Science, Ajloun College, \\ Al-Balqa Applied University \\ JORDAN \\ AMANI SHEIMAT \\ Department of Basic Sciences and Mathematics \\ Faculty of Sciences, Philadelphia University \\ JORDAN \\ ABDULAZEEZ ALKOURI \\ Department of Mathematics, Science college \\ Ajloun National University \\ JORDAN
}

\begin{abstract}
Complex fuzzy sets (CFS) generalize traditional fuzzy sets (FS) since the membership functions of CFS reduces to the membership functions of FS. FS values are always at $[0,1]$, unlike CFS which has values in the unit disk of $\mathrm{C}$. This paper merges notion and concept in group theory and presents the notion of a complex fuzzy subgroup of a group. This proposed idea represents a more general and better optional mathematical tool as one of the approaches in the fuzzy group. However, this research defines the notion of complex fuzzy subgroupiod, complex fuzzy normal subgroup, and complex fuzzy left(right) ideal. Therefore, the lattice, homomorphic preimage, and image of complex fuzzy subgroupiod and ideal are introduced and studied its properties. Finally, complex fuzzy subgroups and their properties are presented and investigated.
\end{abstract}

Key-Words: - Fuzzy sets, fuzzy subgroups and groups, fuzzy subgroupoid and ideal, complex fuzzy subgroupoid and ideal, Complex fuzzy sets, fuzzy subgroups.

Received: March 13, 2021. Revised: July 16, 2021. Accepted: July 31, 2021. Published: September 4, 2021.

\section{Introduction}

In 1965, the fuzzy set was presented by Zadeh [1] and become a vigorous area of research in graph theory, number theory, probability, statistic, topology and measure theory etc. Also, Fuzzy theory was employed in several fields to solve real life problems such as classification, optimization, pattern recognition and others see [2] - [5]. The notion of fuzzy subgruop was presented by Rosenfeld [6] and this concept leads to establish many important properties. The fuzzy subgroup has been generalized by J. M. Anthony and H. Sherwood in [7]. After that, in 1981 $\mathrm{Wu}[8]$ discussed fuzzy normal subgroups. The fuzzy coset concept was studied by N. P. Makherjee and
Bhattacharya [9]. Murali and Makamba [10], [11] introduced fuzzy subgroup, abelian group.

Numerous studies have been conducted in [12] - [23] with the concept of extending the co-domain of membership function from $[0,1]$ to be the closed unit disk which is defined in $\mathbb{C}$. Henc, the co-domain of Complex fuzzy set (CFS) will be presented as $\{a|a \in \mathbb{C}| a \mid, \leq 1\}$.

The CFS values are known to be shrink to fuzzy set without the phase membership $\nu(p)$ [18]. Thus, CFS advanced acts as additional members. The generalized notion of CFS represents two semantics 
"uncertainty and periodicity information" concurrently. Indeed level of comparable related data is disclosed by using phase degree while detecting different meanings in a different phase. The complex numbers $\ell(p) e^{i \nu(p)}$ can represent the uncertainty and periodicity semantics by amplitude $\ell(p)$ and phase $\nu(p)$ terms. These terms have values in $[0,1]$. In this research, the value $\alpha \in(0, n \pi]$, this value represents the periodicity in complex numbers could apply in $n$ levels not only in one level of the unit disk as in [24]. As a comparative study in the field of Algebra. There are two approaches appeared in the literature. First approach given by the innovators Rosenfeld [6]. He made a wonderful work to defeat the obstacles appears from the absence of fuzzy universal set. Other approach is constructed by Dib [25] in a natural way. Dib created the notion of fuzzy space to be switched the notion of universal set in the traditional case. Therefore, the fuzzy group is presented by satisfying the usual conditions of the group depends to define a fuzzy binary operation on a fuzzy space $(X, I)$. The fuzzy subgroup of Rosenfeld, Anthony and Sherwood [7] included in Dib's approach with some restrictions, see [25]. Recently Alsarahead and Ahmad [24] implemented the idea of combining complex fuzzy sets in algebra. Also, Alsarahead and Ahmad treated the complex fuzzy subgroups as two components: fuzzy subgroup for the amplitude term with values lies in $[0,1]$ and $\pi$-fuzzy subgroup for the phase term lies in $[0,2 \pi]$.

Some researchers established and studied propositional complex fuzzy logic [18], which resolves some constraints in complex fuzzy logic theory. Part of manipulations of CFS and its resolution initiate axiomatic for propositional complex fuzzy logic [20] - [23].

A complex fuzzy group is considered as a generalization of Fuzzy groups. The inherent application that appears in the fuzzy group can be used by a complex fuzzy group with the ability to enhance the structure to adapt the periodicity semantics. In general, group theory and fuzzy group theory has been used in general applications in medical diagnosis, pattern recognition and and classification of knowledges [26] - [30]. This research aims to build the basic structure of $\mathrm{CF}$ group and to present its properties. As future research we will use CF group to represent several applications in the field of medical diagnosis, pattern recognition, and classification knowledge by highlighting and considering a periodic information.

In this research, we combine two notions of CFS and theory of groups to build a new concept called a complex fuzzy subgroup of a group in more general and details than reference [24]. We represent both the amplitude and phase term by fuzzy sets with ranges of values lies in $[0,1]$. However, this research begins by defining the notion of complex fuzzy subgroupiod, normal subgroup, and left(right) ideal. Therefore, the lattice, homomorphic input(pre-image), and output(image) of complex fuzzy subgroupiod and ideal are introduced and studied its properties. Finally, complex fuzzy subgroups and their properties are presented and investigated.

\section{Preliminaries}

Definition 1. [I] Let $\mathbb{V}$ be a non empty set. A fuzzy set is just a function $\psi: \mathbb{V} \rightarrow[0,1]$.

Definition 2. [18] Assume that the set $M$ is a complex fuzzy and it is defined on $P$ a universe of discourse. Then it is characterized by it membership function $\psi_{M}(p), \psi_{M}(p)=\ell_{M}(p) e^{i \nu_{M}(p)}$, where $i=\sqrt{-1}$, $\ell_{M}(p) \in[0,1]$ and $\nu_{M}(p) \in \mathbb{R}$. So that, the CFS $M=\left\{\left(p, \psi_{M}(p)\right): p \in P\right\}$.

Definition 3. [18] Following is a representation of a complex fuzzy complement of $M$ :

$\bar{M}=\left\{\left(p, \psi_{\bar{M}}(p)\right): p \in P\right\}=\left\{\left(p, \ell_{\bar{M}}(p) e^{i \nu_{\bar{M}}(p)}\right):\right.$ $p \in P\}$, where $\ell_{\bar{M}}(p)=1-\ell_{M}(p)$ and $\nu_{\bar{M}}(p)=$ $\nu_{M}(p), \nu_{\bar{M}}(p)=2 \pi-\nu_{M}(p)$, or $\nu_{\bar{M}}(p)=\pi+\nu_{M}(p)$

Definition 4. [31] Let $\psi_{M}(p)=\ell_{M}(p) e^{i \nu_{M}(p)}$ and $\psi_{N}(p)=\ell_{N}(p) e^{i \nu_{N}(p)}$ be two membership functions of complex fuzzy sets $M$ and $N$ respectively, on $P$. It indicates that $M$ is greater than $N$, denoted by $M \supseteq$ $N$ or $N \subseteq M$, if for any $p \in P, \ell_{M}(p) \leq \ell_{N}(p)$ and $\nu_{M}(p) \leq \nu_{N}(p)$.

Definition 5. [18] Union of two complex fuzzy sets of $M$ and $N$, denoted $M \cup N$, is a function

$$
\begin{gathered}
U:\{x|x \in \mathbb{C},| x \mid \leq 1\} *\{y|y \in \mathbb{C},| y \mid \leq 1\} \\
\rightarrow\{k|k \in \mathbb{C},| k \mid \leq 1\}
\end{gathered}
$$

where, $U\left(\psi_{M}(p), \psi_{N}(p)\right)=\psi_{M \cup N}(p) ; \forall p \in P$.

The function $U$ must satisfy the following axiomatic, for any $x, y, r, k \in\{p|p \in \mathbb{C}| p \mid, \leq 1\}$

A1. $U(x, 0)=x$.

A2. $|y| \leq|k| \Rightarrow|U(x, y)| \leq|U(x, k)|$.

A3. $U(x, y)=U(y, x)$.

A4. $U(x, U(y, k))=U(U(x, y), k)$.

A5. $U$ is a continuous function.

A6. $|U(x, x)|>|x|$.

A7. $|x| \leq|r|$ and $|y| \leq|k| \Rightarrow|U(x, y)| \leq|U(r, k)|$.

Definition 6. [18] Intersection between $M$ and $N$ in complex fuzzy set is denoted by using $M \cap N$, as following explanation:

$$
I:\{x|x \in \mathbb{C},| x \mid \leq 1\} *\{y|y \in \mathbb{C},| y \mid \leq 1\}
$$




$$
\rightarrow\{k|k \in \mathbb{C},| k \mid \leq 1\}
$$

where, $I\left(\psi_{M}(p), \psi_{N}(p)\right)=\psi_{M \cap N}(p) ; \forall p \in P$.

The function $I$ must satisfy at least the following axiomatic requirements, for any $x, y, r, k \in\{p|p \in \mathbb{C}| p \mid, \leq 1\}$

A1. $|y|=1$, if $I(x, y)=|x|$.

A2. $|y| \leq|k| \Rightarrow|I(x, y)| \leq|I(x, k)|$.

A3. $I(x, y)=I(y, x)$.

A4. $I(x, I(y, k))=I(I(x, y), k)$.

A5. $I$ is a continuous function.

A6. $|I(x, x)|<|x|$.

A7. $|x| \leq|r|$ and $|y| \leq|k| \Rightarrow|I(x, y)| \leq|I(r, k)|$.

Following are the example of $s$-norms and $t$ norms. The universe of discourse $\mathbb{V}$ include any two of CFSs $M$ and $N$.

$$
\begin{aligned}
& \text { s-norm. } \\
& \text { Basic s-norm: } \\
& M \cup N=\left\{\left(p, \max \left(\ell_{M}(p), \ell_{N}(p)\right)\right.\right. \\
& \left.\left.e^{i \alpha \max \left(\nu_{M}(p), \nu_{N}(p)\right)}\right): p \in P\right\} \\
& \text { Yager } S \text { - norm: } \\
& s\left(\left(x e^{\dot{x}}\right),\left(y e^{\dot{y}}\right)\right)=s\left(s_{\bar{w}}^{\ell}(x, y), s_{\bar{w}}^{w}(\dot{x}, \hat{y})\right) \\
& \text { where } s_{\bar{w}}^{\ell}(x, y)= \\
& \min \left(1,\left(x^{\bar{w}}+y^{\bar{w}}\right)^{1 / \bar{w}}\right) \\
& \text { and } s_{\bar{w}}^{w}(\dot{x}, \dot{y})=1-\min \left(1,\left((1-\dot{x})^{\bar{w}}+\right.\right. \\
& \left.\left.\left(1-y^{\prime}\right)^{\bar{w}}\right)^{1 / \bar{w}}\right) \text { with } \bar{w} \in(0, \infty)
\end{aligned}
$$

\section{t-norm.}

Definition 7. [6] For a group $H$, a fuzzy subgroup $\psi$ is fuzzy subset of $H$ which is satisfy:

$$
\begin{aligned}
& \text { 1. } \psi(p q) \geq \min \{\psi(p), \psi(q)\} \\
& \text { 2. } \psi\left(p^{-1}\right) \geq \psi(p) ; \forall p, q \in H
\end{aligned}
$$

Definition 8. [8] If $\psi\left(p^{-1} q p\right) \geq \psi(q) ; \forall p, q \in H$, then the fuzzy subgroup $\psi$ is normal fuzzy subgroup of a group $H$.

\section{Complex Fuzzy Subgroupoids, Normal and Ideals}

Let a closed binary composition (multiplicatively) under a set $M$ be a groupoid. We recall that a function mapping $M$ into unit disk, $[0,1] e^{i \alpha[0,1]}$, is named a complex fuzzy set. The scaling factor $\alpha \in(0, n \pi]$, is employed to narrate the characteristics of the phases lies in the unit disk and $(0, n \pi]$. Thus, the term of phase may literally explain the fuzzy information, and their values belong to $[0,1]$.

Definition 9. Let $\psi$ be a complex fuzzy subgroupoid of $M$ if, $\forall p, q \in M, \psi(p q)=\ell(p q) e^{i \alpha \theta(p q)}$ $\geq \min (\ell(p), \ell(q)) e^{i \alpha \min (\theta(p), \theta(q))}$ or equivalent to $\ell(p q) \geq \min (\ell(p), \ell(q))$ and $\theta(p q) \geq$ $\min (\theta(p), \bar{\theta}(q))$.

If $\psi(p q)=\ell(p q) e^{i \alpha \theta(p q)} \geq \ell(q) e^{i \alpha \theta(q)}$, then $\psi$ named a complex fuzzy left ideal, if $\psi(p q)=$ $\ell(p q) e^{i \alpha \theta(p q)} \geq \ell(p) e^{i \alpha \theta(p)}$, then $\psi$ named a complex fuzzy right ideal, if it is a complex fuzzy ideal (satisfied both: left and right) (or equivalently: if $\ell(p q) \geq$ $\max (\ell(p), \ell(q))$ and $\theta(p q) \geq \max (\theta(p), \theta(q)))$.

Moreover, one can prove that complex fuzzy ideal is a complex fuzzy subgroupoid and for any of the complex fuzzy subgroupoid in $M$, we get that $\psi\left(p^{n}\right)=\ell\left(p^{n}\right) e^{i \alpha \theta\left(p^{n}\right)} \geq \ell(p) e^{i \alpha \theta(p)}, \forall p \in M$, where $p^{n}$ is any composite of $p$ 's.

Proposition 1. For any $\varphi \in[0,1] e^{i \alpha[0,1]},\{z \mid z \in$ $\left.M ; \psi(z)=\ell(z) e^{i \alpha \theta(z)} \geq \varphi\right\}$, is a right(left) ideal or subgroupoid, if $\psi$ is a complex fuzzy right(left) ideal or subgroupoid.

Theorem 1. Let $\psi$ be assumed $\{0,1\} e^{i \alpha\{0,1\}}$, in this $\psi$ function as characteristic function of $N \subset M$. Then $\psi$ is a complex fuzzy right(left) ideal or subgroupoid if and only if $N$ is a right(left) ideal or subgroupoid, respectively

Proof. If $\psi$ is into $\{0,1\} e^{i \alpha\{0,1\}}$ then $\ell(p q) \geq$ $\min (\ell(p), \ell(q))$ and $\theta(p q) \geq \min (\theta(p), \theta(q))$, is equivalent to $\ell(p)=\ell(q)=0$ or 1 and $\theta(p)=\theta(q)=0$ or 1 . So that, we have four cases:

Case $1 \ell(p q)=1$ and $\theta(p q)=1^{\prime \prime}$.

Case $2 \ell(p q)=1$ and $\theta(p q)=0 "$.

Case $3 \ell(p q)=0$ and $\theta(p q)=1 "$.

Case $4 \ell(p q)=0$ and $\theta(p q)=0 "$.

Similarly, $\ell(p q) \geq \ell(q)$ and $\theta(p q) \geq \theta(q)$ or $" q$ in $N$ indicates $p q$ in $N$.

For the rest of article, the $\varphi_{N}$, is the characteristic function of $N$.

Theorem 2. Let $\psi=\ell e^{i \alpha \theta}$ be a complex fuzzy subgroup of a group $H$, then for any $p, q \in H$. If $\psi(p q)=\ell(p q) e^{i \alpha \theta(p q)}=\ell(e) e^{i \alpha \theta(e)}=\psi(e)$, then $\psi(p)=\ell(p) e^{i \alpha \theta(p)}=\ell(q) e^{i \alpha \theta(q)}=\psi(q)$. 
Proof. Let $\psi=\ell e^{i \alpha \theta}$ is complex fuzzy subgroup, according to Definition 9

$$
\begin{aligned}
\psi(p) & =\ell(p) e^{i \alpha \theta(p)} \\
& =\ell\left(p q q^{-1}\right) e^{i \alpha \theta\left(p q q^{-1}\right)} \\
& =\ell\left((p q) q^{-1}\right) e^{i \alpha \theta\left((p q) q^{-1}\right)} \\
& \geq \min \left(\ell(p q), \ell\left(q^{-1}\right)\right) e^{i \alpha \min \left(\theta(p q), \theta\left(q^{-1}\right)\right)} \\
& =\min (\ell(e), \ell(q)) e^{i \alpha \min (\theta(e), \theta(q))} \\
& =\ell(q) e^{i \alpha \theta(q)} \\
& =\psi(q)
\end{aligned}
$$

Then $\psi(p)=\ell(p) e^{i \alpha \theta(p)} \geq \ell(q) e^{i \alpha \theta(q)}=\psi(q)$.

On the other side,

$$
\begin{aligned}
\psi(q) & =\ell(q) e^{i \alpha \theta(q)} \\
& =\ell\left(p^{-1} p q\right) e^{i \alpha \theta\left(p^{-1} p q\right)} \\
& =\ell\left(p^{-1}(p q)\right) e^{i \alpha \theta\left(p^{-1}(p q)\right)} \\
& \geq \min \left(\ell\left(p^{-1}\right), \ell(p q)\right) e^{i \alpha \min \left(\theta\left(p^{-1}\right), \theta(p q)\right)} \\
& =\min (\ell(p), \ell(e)) e^{i \alpha \min (\theta(p), \theta(e))} \\
& =\ell(p) e^{i \alpha \theta(p)} \\
& =\psi(p)
\end{aligned}
$$

Then $\psi(p)=\ell(p) e^{i \alpha \theta(p)} \leq \ell(q) e^{i \alpha \theta(q)}=\psi(q)$.

Thus $\psi(p)=\ell(p) e^{i \alpha \theta(p)}=\ell(q) e^{i \alpha \theta(q)}=\psi(q)$.

Definition 10. Assume $H$ is a group and $\psi=\ell e^{i \alpha \theta}$ is complex fuzzy subgroup of $H$, therefore $\psi=\ell e^{i \alpha \theta}$ is named complex normal fuzzy subgroup of $H$ if $\psi\left(p^{-1} q p\right)=\ell\left(p^{-1} q p\right) e^{i \alpha \theta\left(p^{-1} q p\right)} \geq \ell(q) e^{i \alpha \theta(q)}=$ $\psi(q) \forall p, q \in H$.

Corollary 1. Assume $H$ is a group and $\psi=\ell e^{i \alpha \theta}$ is a complex fuzzy subgroup of $H$, therefore the three proceeding statements are equivalent:

1. $\psi\left(p q p^{-1}\right)=\ell\left(p q p^{-1}\right) e^{i \alpha \theta\left(p q p^{-1}\right)} \geq$ $\ell(q) e^{i \alpha \theta(q)}=\psi(q) \forall p, q \in H$

2. $\psi\left(p q p^{-1}\right)=\ell\left(p q p^{-1}\right) e^{i \alpha \theta\left(p q p^{-1}\right)}=$ $\ell(q) e^{i \alpha \theta(q)}=\psi(q) \forall p, q \in H$

3. $\psi(p q)=\ell(p q) e^{i \alpha \theta(p q)}=\ell(q p) e^{i \alpha \theta(q p)}=\psi(q p)$ $\forall p, q \in H$

(i.e. $\psi$ is complex normal fuzzy subgroup)

Proof. Straightforward.

Theorem 3. Assume $H$ is a group and $\psi$ is a complex fuzzy subgroup of $H$. So that, $\psi$ is a complex normal fuzzy subgroup iff $\psi\left(p q(q p)^{-1}\right)=$ $\ell\left(p q(q p)^{-1}\right) e^{i \alpha \theta\left(p q(q p)^{-1}\right)} \geq \ell(q) e^{i \alpha \theta(q)} \forall p, q \in H$.
Proof. Let $\psi$ be a complex normal fuzzy subgroup, let $p, q \in H$ then $\psi\left(p q p^{-1} q^{-1}\right)$

$$
\begin{aligned}
& =\ell\left(p q p^{-1} q^{-1}\right) e^{i \alpha \theta\left(p q p^{-1} q^{-1}\right)} \\
& \geq \min \left(\ell\left(p q p^{-1}\right), \ell\left(q^{-1}\right)\right) e^{i \alpha \min \left(\theta\left(p q p^{-1}\right), \theta\left(q^{-1}\right)\right)} \\
& =\min (\ell(q), \ell(q)) e^{i \alpha \min (\theta(q), \theta(q))} \\
& =\min (\psi(q), \psi(q)) \\
& =\psi(q) .
\end{aligned}
$$

Therefore $\psi\left(p q(q p)^{-1}\right)=\ell\left(p q(q p)^{-1}\right) e^{i \alpha \theta\left(p q(q p)^{-1}\right)}$ $\geq \ell(q) e^{i \alpha \theta(q)}=\psi(q)$

Conversely, let $p, q \in H$ and $\psi\left(p q(q p)^{-1}\right)=$ $\ell\left(p q(q p)^{-1}\right) e^{i \alpha \theta\left(p q(q p)^{-1}\right)} \geq \ell(q) e^{i \alpha \theta(q)}$, then $\psi\left(p q p^{-1}\right)$

$$
\begin{aligned}
& =\ell\left(p q p^{-1}\right) e^{i \alpha \theta\left(p q p^{-1}\right)} \\
& =\psi\left(p q p^{-1} q^{-1} q\right)=\ell\left(p q p^{-1} q^{-1} q\right) e^{i \alpha \theta\left(p q p^{-1} q^{-1} q\right)} \\
& \geq \min \left(\ell\left(p q p^{-1} q^{-1}\right), \ell(q)\right) e^{i \alpha \min \left(\theta\left(p q p^{-1} q^{-1}\right), \theta(q)\right)} \\
& \geq \min (\ell(q), \ell(q)) e^{i \alpha \min (\theta(q), \theta(q))} \\
& =\ell(q) e^{i \alpha \theta(q)} \\
& =\psi(q) \quad \forall p, q \in H .
\end{aligned}
$$

Then $H$ has a complex normal fuzzy subgroup, denoted by $\psi$.

Theorem 4. Let $\psi$ be a complex fuzzy subgroup of $H$. If $\psi\left(p^{2}\right)=\ell\left(p^{2}\right) e^{i \alpha \theta\left(p^{2}\right)}=\ell(e) e^{i \alpha \theta(e)}=\psi(e)$ $\forall p, q \in H$. Then $\psi$ is a complex normal fuzzy subgroup.

Proof. Let $p, q \in H, \psi$ be a complex fuzzy subgroup of $H$ and $\psi\left(p^{2}\right)=\ell\left(p^{2}\right) e^{i \alpha \theta\left(p^{2}\right)}=\ell(e) e^{i \alpha \theta(e)}=$ $\psi(e)$, then we have:

$$
\begin{aligned}
\ell(e) e^{i \alpha \theta(e)} & =\psi(e) \\
& =\psi\left((p q)^{2}\right) \\
& =\psi(p q p q) \\
& =\psi\left(\left(p q p^{-1}\right)\left(p^{2} q\right)\right) \\
& =\ell\left(\left(p q p^{-1}\right)\left(p^{2} q\right)\right) e^{i \alpha \theta\left(\left(p q p^{-1}\right)\left(p^{2} q\right)\right)}
\end{aligned}
$$

Thus it follows, by Theorem 2 .

$$
\begin{aligned}
\psi\left(p^{-1} q p\right) & =\ell\left(p^{-1} q p\right) e^{i \alpha \theta\left(p^{-1} q p\right)} \\
& =\ell\left(p^{2} q\right) e^{i \alpha \theta\left(p^{2} q\right)} \\
& \geq \min \left(\ell\left(p^{2}\right) e^{i \alpha \theta\left(p^{2}\right)}, \ell(q) e^{i \alpha \theta(q)}\right) \\
& \geq \min \left(\ell(e) e^{i \alpha \theta(e)}, \ell(q) e^{i \alpha \theta(q)}\right) \\
& =\min (\psi(e), \psi(q)) \\
& =\psi(q) .
\end{aligned}
$$

Therefore, $\psi\left(p^{-1} q p\right) \geq \psi(q)$ and hence $\psi$ is a complex normal fuzzy subgroup. by:

Note that, the inclusion of CFS in $M$ was given $\ell(p) e^{i \alpha \theta(p)} \leq k(p) e^{i \alpha \omega(p)}$, equivalent to $\ell(p) \leq k(p)$, 
and $\theta(p) \leq \omega(p) \forall p \in M$.

Let the supremum $\cup$ and infimum $\cap$, respectively. In CFS ordering, the set of all complex fuzzy sets in $M$ is a complete lattice $\chi$. The greatest element of $\chi$ is $1 e^{i \alpha(1)}=\varphi_{M}$, and the least element of $\chi$ is $0 e^{i \alpha(0)}$ $=\varphi_{\phi}$, which both are constant functions. Hence, $\varphi_{M}$ and $\varphi_{\phi}$ are complex fuzzy ideals subgroupoids.

Theorem 5. Infimum $\cap$ of complex fuzzy subgroupoids are complex fuzzy subgroupoid.

$$
\begin{aligned}
& \text { Proof. } \cap \psi_{i}(p q)=\cap \ell_{i}(p q) e^{i \alpha \cap \theta_{i}(p q)} \\
& \quad=\inf \left[\ell_{i}(p q)\right] e^{i \alpha \inf \left[\theta_{i}(p q)\right]} \\
& \geq \inf \left[\min \left\{\ell_{i}(p), \ell_{i}(q)\right\}\right] e^{i \alpha \inf \left[\min \left\{\theta_{i}(p), \theta_{i}(q)\right\}\right]} \\
& =\min \left\{\inf \ell_{i}(p), \inf \ell_{i}(q)\right\} e^{i \alpha \min \left\{\inf \theta_{i}(p), \inf \theta_{i}(q)\right\}} \\
& \quad=\min \left\{\cap \ell_{i}(p), \cap \ell_{i}(q)\right\} e^{i \alpha \min \left\{\cap \theta_{i}(p), \cap \theta_{i}(q)\right\}} .
\end{aligned}
$$

The infimum of $\psi_{i}=\ell_{i} e^{i \alpha \theta_{i}}$ is $\cap \psi_{i}=\cap \ell_{i} e^{i \alpha \cap \theta_{i}}$, while their supremum is the $\cup$ of all $\psi_{i^{\prime} s}$.

Definition 11. The complex fuzzy subgroupoid $(\gamma=$ $\left.k e^{i \alpha \omega}\right)$ generated by $\gamma$ is defined as the least complex fuzzy subgroupoid which $\supseteq \gamma$.

Proposition 2. $\left(\varphi_{N}\right)=\varphi_{N}$, where $N$ generates the subgroupoid of $(N)$.

Proof. Let $\psi=\ell e^{i \alpha \theta} \supseteq \varphi_{(N)}$, we have $\psi=1 e^{i \alpha(1)}$ (i.e. $\ell=1$ and $\theta=1) \forall p \in N$; since $\psi=1 e^{i \alpha(1)}$ is a complex fuzzy subgroupoid, then we have $\psi=$ $1 e^{i \alpha(1)}$ for any composite of elements of $N$. Therefore, $\psi=\ell e^{i \alpha \theta} \supseteq \varphi_{(N)}$. Thus $\varphi_{(N)} \subseteq$ the infimum $\cap$ of all such $\psi_{i^{\prime} s}$. In reverse, by using Proposition 1 we have $\varphi_{(N)}$ equal $\psi$.

Therefore, the lattice of $N$ in a sublattice, of the complex fuzzy subgroupoid, can be consider the subgroupoid lattice of $N$.

Proposition 3. Both infimum $\cap$ and supremum $\cup$, for all set of complex fuzzy right(left) ideals, is a complex fuzzy right(left) ideal.

$$
\begin{aligned}
& \text { Proof. } \cap \psi_{j}(p q)=\left[\cap \ell_{j}(p q)\right] e^{i \alpha\left[\cap \theta_{j}(p q)\right]} \\
& =\inf \left[\ell_{j}(p q)\right] e^{i \alpha \inf \left[\theta_{j}(p q)\right]} \\
& =\inf \left[\ell_{j}(q)\right] e^{i \alpha \inf \left[\theta_{j}(q)\right]}=\left[\cap \ell_{j}(q)\right] e^{i \alpha\left[\cap \theta_{j}(q)\right]} .
\end{aligned}
$$$$
\text { Likewise, in the same way for supremum } \cup \text { and on }
$$
the right ideal.

\section{Homomorphic input(pre-image) and output(image) of Complex Fuzzy Subgroupoids and Ideals}

Let $\psi=\ell e^{i \alpha \theta}$ be a complex fuzzy set in $M$, and let $g$ be a function defined on $M$, so the CFS $f$ in $g(M)$ is defined by:

$$
f(q)=k(q) e^{i \alpha \omega(q)}=\left[\sup _{p \in g^{-1}(q)} k(p)\right] e^{i \alpha\left[\sup _{p \in g^{-1}(q)} \omega(p)\right]}
$$

under $g$ all $q \in g(M)$, is considered as the output of $\psi=\ell e^{i \alpha \theta}$ respect to $g$. Likewise, if $f$ is a CFS in $g(M)$, then the CFS $\psi=g o f=g(f)=g\left(g^{-1}(\psi)\right)$ in $M$ (In other word, the CFS is defined by $\psi(p)=$ $f(g(p)) \forall p \in M)$ is named the input of $f$ respect to $g$. Also, if $\psi=\varphi_{M}$, then the output of $\psi$ respect to $g$ is just $\varphi_{g(M)}$; and if $f=\varphi_{W}$ (such that $W \subseteq g(M)$ ), then the input of $f$ respect to $g$ is just $\varphi_{g^{-1}}(W)$.

Theorem 6. A homomorphic input of a complex fuzzy subgroupoid is a complex fuzzy subgroupoid. Likewise, A homomorphic input of a left(right) ideal is a complex fuzzy subgroupoid.

$$
\begin{aligned}
& \text { Proof. } \psi(p q)=\ell(p q) e^{i \alpha \theta(p q)} \\
& \qquad f(g(p q))=k(g(p q)) e^{i \alpha \omega(g(p q))} \\
& =k(g(p) g(q)) e^{i \alpha \omega(g(p) g(q))} \\
& \geq \min \{k(g(p)), k(g(q))\} e^{i \alpha \min \{\omega(g(p)), \omega(g(q))\}} \\
& =\min \{\ell(p), \ell(q)\} e^{i \alpha \min \{\theta(p), \theta(q)\}} \\
& =\min \{\psi(p), \psi(q)\},
\end{aligned}
$$

also ideals will have similar structure.

A CFS $\psi=\ell e^{i \alpha \theta} \in M$ has the supremum property if, $N \subseteq M$, has $n_{0} \in N$ such as $\psi\left(n_{0}\right)=$ $\ell\left(n_{0}\right) e^{i \alpha \theta\left(n_{0}\right)}=\left[\sup _{n \in N} \ell(n)\right] e^{i \alpha\left[\sup _{n \in N} \theta(n)\right]}$. For example, if $\psi=\ell e^{i \alpha \theta}(\ell$ and $\theta)$ can only take any finite values (particularly, when it has a characteristic function), and has the supremum property.

Theorem 7. A homomorphic output of a complex fuzzy subgroupoid, satisfied the supremum property, is also a complex fuzzy subgroupoid, in like manner for the left(right) ideal.

Proof. Let $g(p), g(q) \in g(M), p_{0} \in g^{-1}(g(p))$, $q_{0} \in g^{-1}(g(q))$.

Then $\psi\left(p_{0}\right)=\ell\left(p_{0}\right) e^{i \alpha \theta\left(p_{0}\right)}=$

$$
\begin{aligned}
& {\left[\sup _{n \in g^{-1}(g(p))} \ell(n)\right] e^{i \alpha\left[\sup _{n \in g^{-1}(g(p))} \theta(n)\right]}, } \\
\psi\left(q_{0}\right)= & \ell\left(q_{0}\right) e^{i \alpha \theta\left(q_{0}\right)}= \\
& {\left[\sup _{n \in g^{-1}(g(q))} \ell(n)\right] e^{i \alpha\left[\sup _{n \in g^{-1}(g(q))} \theta(n)\right]}, }
\end{aligned}
$$


respectively.

Then $f(g(p) g(q))$

$$
\begin{aligned}
& =\sup _{Z \in g^{-1}(g(p) g(q))} \ell(Z) e^{i \alpha \theta(Z)} \\
& \geq \min \left\{\ell\left(p_{0}\right), \ell\left(q_{0}\right)\right\} e^{i \alpha \min \left\{\theta\left(p_{0}\right), \theta\left(q_{0}\right)\right\}} \\
& =\min \{k(g(p)), k(g(q))\} e^{i \alpha \min \{\omega(g(p)), \omega(g(q))\}} \\
& =\min \{f(g(p)), f(g(q))\},
\end{aligned}
$$

also ideals will have similar structure.

Let any function $g$ be defined on $M$ and $f \in g(M)$ is a CFS, then the output of the input of $f$ respect to $g$ equal $f$.

$$
\begin{gathered}
\sup _{p \in g^{-1}(q)} f(g(p))=\sup _{p \in g^{-1}(q)} k(g(p)) e^{i \alpha\left[\sup _{p \in g^{-1}(q)} \omega(g(p))\right]} \\
=k(q) e^{i \alpha \omega(q)}=f(q), \forall q \in g(M) .
\end{gathered}
$$

Conversely, if $\psi=\ell e^{i \alpha \theta}$ is a CFS in $M$, then the input of the output of $\psi=\ell e^{i \alpha \theta}$ respect to $g$ always $\supseteq \psi=\ell e^{i \alpha \theta}$, since

$$
\begin{gathered}
\sup _{Z \in g^{-1}(g(p) g(q))} \psi(Z)=\sup _{Z \in g^{-1}(g(f) g(q))} \ell(Z) e^{i \alpha \theta(Z)} \geq \\
\ell(p) e^{i \alpha \theta(f)}=\psi(f), \forall p \in M .
\end{gathered}
$$

We call $\psi=\ell e^{i \alpha \theta} g$-invariant if $g(p)=g(q) \Rightarrow$ $\psi(p)=\ell(p) e^{i \alpha \theta(p)}=\ell(q) e^{i \alpha \theta(q)}=\psi(q)$. Clearly if $\psi=\ell e^{i \alpha \theta}$ is $g$-invariant, then the input of its output respect to $g$ is $\psi=\ell e^{i \alpha \theta}$ itself.

\section{Complex Fuzzy Subgroups}

Definition 12. Let $M$ be a group, a complex fuzzy subgroupoid $\psi=\ell e^{i \alpha \theta}$ of $M$ can be named a complex fuzzy subgroup of $M$ if $\psi\left(p^{-1}\right)=\ell\left(p^{-1}\right) e^{i \alpha \theta\left(p^{-1}\right)}$ $\geq \ell(p) e^{i \alpha \theta(p)}=\psi(p)$, (i.e. $\ell\left(p^{-1}\right) \geq \ell(p)$ and $\left.\bar{\theta}\left(p^{-1}\right) \geq \theta(p)\right) \forall p \in M$.

The scaling factor $\alpha \in(0, n \pi]$, is employed to confine the characteristics of the phases lies in the unit disk and $(0, n \pi]$. Thus, the term of phase may literally explain the fuzzy information, and their values belong to $[0,1]$.

Proposition 4. $N$ is a subgroup iff $\varphi_{N}$ is a complex fuzzy subgroup.

Proposition 5. For the infimum $\cap$ of complex fuzzy subgroups, is also a complex fuzzy subgroup.
Proposition 6. Complex fuzzy subgroup can be taken from the characteristic function of a set, where a set is generator of the characteristic function of a subgroup.

Proposition 7. Let $\psi=\ell e^{i \alpha \theta}$ be a complex fuzzy subgroup of $M$, then $\psi\left(p^{-1}\right)=\ell\left(p^{-1}\right) e^{i \alpha \theta\left(p^{-1}\right)}$ $=\ell(p) e^{i \alpha \theta(p)}=\psi(p)$ and $\psi(p)=\ell(p) e^{i \alpha \theta(p)} \leq$ $\ell(e) e^{i \alpha \theta(e)}=\psi(e), \forall p \in M$, the identity element of $M$ is denoted by $e$.

$$
\begin{aligned}
& \text { Proof. } \psi(p) \quad=\quad \ell(p) e^{i \alpha \theta(p)}= \\
& \ell\left(\left(p^{-1}\right)^{-1}\right) e^{i \alpha \theta\left(\left(p^{-1}\right)^{-1}\right)} \geq \ell\left(p^{-1}\right) e^{i \alpha \theta\left(p^{-1}\right)} \geq \\
& \ell(p) \quad e^{i \alpha \theta(p)}, \quad \text { hence } \quad \psi(e)= \\
& \ell(e) e^{i \alpha \theta(e)}=\quad \ell\left(p p^{-1}\right) e^{i \alpha \theta\left(p p^{-1}\right)} \geq \\
& \min \left(\ell(p), \ell\left(p^{-1}\right)\right) e^{i \alpha \min \left(\theta(p), \theta\left(p^{-1}\right)\right)}=\ell(p) e^{i \alpha \theta(p)}=
\end{aligned}
$$

Corollary 2. Let $H_{\psi}=\left\{p: \psi(p)=\ell(p) e^{i \alpha \theta(p)}=\right.$ $\left.\ell(e) e^{i \alpha \theta(e)}=\psi(e)\right\}$ is a subgroup.

Proof. Use Proposition 9

Theorem 8. If $\psi\left(p q^{-1}\right)=\ell\left(p q^{-1}\right) e^{i \alpha \theta\left(p q^{-1}\right)}=$ $\ell(e) e^{i \alpha \theta(e)}=\psi(e)$, then $\psi(p)=\ell(p) e^{i \alpha \theta(p)}=$ $\ell(q) e^{i \alpha \theta(q)}=\psi(q)$.

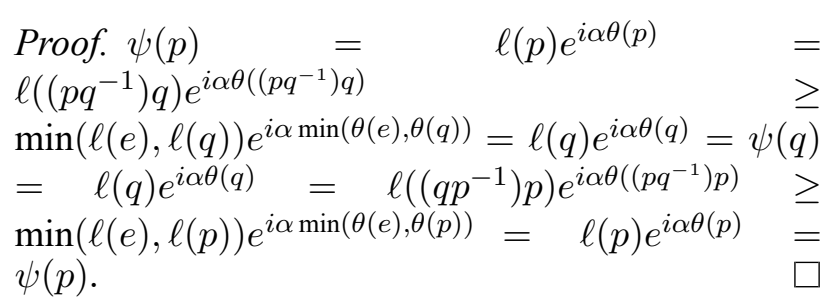

Corollary 3. For each coset of $H_{\psi}, \psi=\ell e^{i \alpha \theta}$ is constant.

Corollary 4. If $H_{\psi}$ has finite index, then $\psi=\ell e^{i \alpha \theta}$ has the supremum property.

Theorem 9. $\psi=\ell e^{i \alpha \theta}$ is a complex fuzzy subgroup of $M$ iff $\psi\left(p q^{-1}\right)=\ell\left(p q^{-1}\right) e^{i \alpha \theta\left(p q^{-1}\right)} \geq$ $\min (\ell(p), \ell(q)) e^{i \alpha \min (\theta(p), \theta(q))}=\min (\psi(p), \psi(q))$ $\forall p, q \in M$.

Proof. Let $\psi=\ell e^{i \alpha \theta}$ be a complex fuzzy subgroup, then $\psi\left(p q^{-1}\right)$

$$
\begin{aligned}
& =\ell\left(p q^{-1}\right) e^{i \alpha \theta\left(p q^{-1}\right)} \\
& \geq \min \left(\ell(p), \ell\left(q^{-1}\right)\right) e^{i \alpha \min \left(\theta(p), \theta\left(q^{-1}\right)\right)} \\
& =\min (\ell(p), \ell(q)) e^{i \alpha \min (\theta(p), \theta(q))} \\
& =\min (\psi(p), \psi(q)) .
\end{aligned}
$$

Conversely, $\psi\left(p q^{-1}\right)=\ell\left(p q^{-1}\right) e^{i \alpha \theta\left(p q^{-1}\right)} \geq$ $\min (\ell(p), \ell(q)) e^{i \alpha \min (\theta(p), \theta(q))}=\min (\psi(p), \psi(q))$, let $q=p$ to obtain $\psi(e)=\ell(e) e^{i \alpha \theta(e)} \geq$ 
$\ell(p) e^{i \alpha \theta(p)}=\psi(p) \forall p \in M$, which leads to, $\psi\left(q^{-1}\right)=\ell\left(e q^{-1}\right) e^{i \alpha \theta\left(e q^{-1}\right)} \geq$

$\min (\ell(e), \ell(q)) e^{i \alpha \min (\theta(e), \theta(q))}=\ell(q) e^{i \alpha \theta(q)}=\psi(q)$, and its follows that $\psi(p q)$

$$
\begin{aligned}
& =\ell(p q) e^{i \alpha \theta(p q)} \\
& =\ell\left(p\left(q^{-1}\right)^{-1}\right) e^{i \alpha \theta\left(p\left(q^{-1}\right)^{-1}\right)} \\
& \geq \min \left(\ell(p), \ell\left(q^{-1}\right)\right) e^{i \alpha \min \left(\theta(p), \theta\left(q^{-1}\right)\right)} \\
& \geq \min (\ell(p), \ell(q)) e^{i \alpha \min (\theta(p), \theta(q))} \\
& =\min (\psi(p), \psi(q))
\end{aligned}
$$

Theorem 10. For two proper complex fuzzy subgroups, the supremum of these subgroups cannot be a group.

Proof. Let $\psi(p)=\ell(p) e^{i \alpha \theta_{\ell}(p)}$ and $\eta(p)=$ $k(p) e^{i \alpha \theta_{k}(p)}$ be two complex fuzzy subgroups such that $\ell(p)=1$ or $k(p)=1$ and $\theta_{\ell}(p)=1$ or $\theta_{k}(p)=1 \forall p \in M$. Let $\ell(u)=\theta_{\ell}(u)=1$, both $\ell(v)$ and $\theta_{\ell}(v) \leq 1, k(v)=\theta_{k}(v)=1$, both $k(u)$ and $\theta_{k}(u) \leq 1$.

Moreover, for $u v$, where $\ell(u v)=\theta_{\ell}(u v)=1$ and since $\psi\left(u^{-1}\right)=\ell\left(u^{-1}\right) e^{i \alpha \theta_{\ell}\left(u^{-1}\right)}=1 e^{i \alpha(1)}$, we have $\psi(v)=\ell\left(u^{-1}(u v)\right) e^{i \alpha \theta_{\ell}\left(u^{-1}(u v)\right)} \geq$ $\min \left\{\ell\left(u^{-1}\right), \ell(u v)\right\} e^{i \alpha \min \left\{\theta_{\ell}\left(u^{-1}\right), \theta_{\ell}(u v)\right\}}=1 e^{i \alpha(1)}$, and if we have $k(u v)=\theta_{k}(u v)=1$, then we get a contradiction.

Theorem 11. A homomorphic input or output of a complex fuzzy subgroup is a complex fuzzy subgroup. Proof. For input, $\psi\left(p^{-1}\right)$

$$
\begin{aligned}
& =\ell\left(p^{-1}\right) e^{i \alpha \theta_{\ell}\left(p^{-1}\right)}=\eta\left(g\left(p^{-1}\right)\right) \\
& =k\left(g\left(p^{-1}\right)\right) e^{i \alpha \theta_{k}\left(g\left(p^{-1}\right)\right)} \\
& =k\left(g(p)^{-1}\right) e^{i \alpha \theta_{k}\left(g(p)^{-1}\right)} \\
& \geq k(g(p)) e^{i \alpha \theta_{k}(g(p))} \\
& =\ell(p) e^{i \alpha \theta_{\ell}(p)} \\
& =\psi(p) .
\end{aligned}
$$

For output, given $g(p) \in g(M)$, let $p_{0} \in g^{-1}(g(p))$ be such that

$$
\psi\left(p_{0}\right)=\ell\left(p_{0}\right) e^{i \alpha \theta_{\ell}\left(p_{0}\right)}=\sup _{z \in g^{-1}(g(p))} \ell(z) e^{i \alpha \theta_{\ell}(z)},
$$

then $\eta\left(g\left(p_{0}\right)^{-1}\right)$

$$
\begin{array}{ll}
= & k\left(g\left(p_{0}\right)^{-1}\right) e^{i \alpha \theta_{k}\left(g\left(p_{0}\right)^{-1}\right)} \\
= & \sup \ell(z) e^{i \alpha \theta_{\ell}(z)} \\
\geq & z \in g^{-1}\left(g(p)^{-1}\right) \\
\geq & \ell\left(\left(p_{0}\right)^{-1}\right) e^{i \alpha \theta_{\ell}\left(\left(p_{0}\right)^{-1}\right)} \\
= & \ell\left(p_{0}\right) e^{i \alpha \theta_{\ell}\left(p_{0}\right)} \\
= & k(g(p)) e^{i \alpha \theta_{k}(g(p))} \\
= & \eta(g(p)) .
\end{array}
$$

Example 1. Let $H$ be the Klien four-group, we have: $H=\left\{p, q: p^{2}=q^{2}=(p q)^{2}=e\right\}$, thus the elements of $H$ are e, $p, q, p q$. Let $m_{i}$, where $i=0,1,2$, be three numbers lie in the unit disk such that $m_{0}>m_{1}>$ $m_{2}$.

Define $A: H \rightarrow\{c: c \in \mathbb{C},|c| \leq 1\}$. Clearly, $A$ is a $C F$ subgroup of $H$, the output of $A$ is $\left\{m_{0}, m_{1}, m_{2}\right\}$.

Example 2. Let $G$ be a nontrivial subgroup of a group $H$, such that $G \neq\{e\}$ and $G \neq H$.

Define $\psi: H \rightarrow\{c: c \in \mathbb{C},|c| \leq 1\}$, where $\psi(p)=\left\{\begin{array}{ll}\ell(p) e^{i \alpha \omega(p)} ; & p \in G \\ 0 e^{i \alpha 0} ; & p \notin G .\end{array}\right.$, where $\ell, \omega$ are arbitary number in $[0,1]$, then $A$ is a CF subgroup of $H$.

Case I: Let $p, q \in H$, since $G \leq H$, if $p, q \in G$ then $p q \in G$. So that, $\psi(p q)=\ell e^{i \alpha \bar{\omega}}, \psi(p)=\ell e^{i \alpha \omega}$, and $\psi(q)=\ell e^{i \alpha \omega}$. Thus, $\psi(p q) \geq \min (\psi(p), \psi(q))$ and for $p \in G, \psi(p)=\psi\left(p^{-1}\right)$.

Case II: Let $p \in G, q \notin G$, then $p q \notin G$. So that, $\psi(p)=\ell e^{i \alpha \omega}, \psi(q)=0=\psi(p q)$. Thus, $\psi(p q) \geq \min (\psi(p), \psi(q))$ and $\psi(p)=\psi\left(p^{-1}\right)$ for $p \in G$ or $p \notin G$.

Case III: Let $p, q \notin G$, then pq may or may not belong to G. So that, in both cases $\psi(p q) \geq \min (\psi(p), \psi(q))$ and $\psi(p)=\psi\left(p^{-1}\right)$. Therefore $A$ is a CF subgroup of $H$.

Now, $\operatorname{Im}(A)=\{0,1\}, \psi_{0}=\left\{p: \psi(p) \geq 0 e^{i \alpha 0}\right\}=$ $H$, and $\psi_{\ell^{i \alpha \omega}}=\left\{p: \psi(p) \geq \ell e^{i \alpha \omega}\right\}=G$. Therefore, we get $\psi(e)=\ell e^{i \alpha \omega}$ but $\psi_{\ell e^{i \alpha \omega}}=G$ which is not equal to $\{e\}$.

Theorem 12. In a group, complex fuzzy left(right) ideals are constant complex functions.

Proof. $(\Rightarrow)$ If $\psi=\ell e^{i \alpha \theta}$ is a constant complex function, then $\psi$ is a complex fuzzy ideal, where $\psi(p q)=\ell(p q) e^{i \alpha \theta(p q)}=\ell(p) e^{i \alpha \theta(p)}=\ell(q) e^{i \alpha \theta(q)}$ $\forall p, q \in M$.

$(\Leftarrow)$ Let $\psi=\ell e^{i \alpha \theta}$ be a complex fuzzy left ideal of a group $M$, therefore $\psi(p q)=\ell(p q) e^{i \alpha \theta(p q)} \geq$ $\ell(q) e^{i \alpha \theta(q)}=\psi(q) \forall p, q$. Assume that $q=e$ gives $\psi(p)=\ell(p) e^{i \alpha \theta(p)} \geq \ell(e) e^{i \alpha \theta(e)}=\psi(e)$ $\forall p$, while letting $p=q^{-1}$ gives $\psi(e)=\ell(e) e^{i \alpha \theta(e)}$ $\geq \ell(q) e^{i \alpha \theta(q)}=\psi(q) \forall q$, thus $\psi(p)=\ell(p) e^{i \alpha \theta(p)}$ $=\ell(e) e^{i \alpha \theta(e)}=\psi(e)$ is a constant complex function.

Theorem 13. Let $\psi$ be any complex fuzzy subgroup of $H$ ( $H$ is a cyclic group of order $r$; $r$ is prime), then $\psi(p)=\ell(p) e^{i \alpha \theta(p)}=\psi(1)=\ell(1) e^{i \alpha \theta(1)}<\psi(0)=$ $\ell(0) e^{i \alpha \theta(0)} \forall p \neq 0$ in $H$, and conversely any such $\psi=\ell e^{i \alpha \theta}$ is a complex fuzzy subgroup. 
Proof. $(\Rightarrow)$ For any such $\psi=\ell e^{i \alpha \theta}, \psi(p q)=$ $\ell(p q) e^{i \alpha \theta(p q)}>\min (\ell(p), \ell(q)) e^{i \alpha \min (\theta(p), \theta(q))}$ is directly since $00=0$, and $\psi\left(p^{-1}\right)=\ell\left(p^{-1}\right) e^{i \alpha \theta\left(p^{-1}\right)}$ $\geq \ell(p) e^{i \alpha \theta(p)}=\psi(p)$ is directly, since $-0=0$.

$(\Leftarrow) \forall p \neq 0$ and $q \neq 0$ in $H, p$ is a sum of $q$ 's and $q$ a sum of $p$ 's, therefore $\psi(p)=\ell(p) e^{i \alpha \theta(p)} \geq$ $\psi(q)=\ell(q) e^{i \alpha \theta(q)} \geq \psi(p)=\ell(p) e^{i \alpha \theta(p)}$.

\section{Discussion}

The hypothesis of this research was derived from a combination between Rosenfeld's approach of a fuzzy subgroup of a group [6] and the idea of extending the codomain of membership function from $[0,1]$ to unit disk in $\mathbb{C}[18]$. The generalization of codomain of characteristic function was tracked from $\{0,1\}$ to $[0,1]$ to unit disk in crisp sets, fuzzy sets, and complex fuzzy sets, respectively. We found that fuzzy sets were extensively applied and studied in many fields of mathematics by using different approaches, like fuzzy algebra, fuzzy topology, fuzzy partial differential equations, etc. Also, complex fuzzy sets were traced similar generalization only in uncertainty sets and algebra as appeared in [6], [18] [23], [31]. This research represents the combination of the amplitude and phase terms in the notion of complex fuzzy groups more generally than the notion presented in Ref, [24]. This combination enables a specific representation of repeated behaviors in possible mathematical formula with periodic or seasonal performance, compactly in a single set [24], used two sets to represent complex fuzzy subgroups and used the phase term as a value lies in $(0,2 \pi]$. The advantage of this research, the phase term plays a similar role as the amplitude term by describing the degree of belongingness value lies in $[0,1]$, whereas the phase term may represent information about the periodicity in some periodic data. Information, that happens periodically, carries a different meaning for the same data/measurements. For example, the temperature $20^{\circ} \mathrm{C}$ can be measured in both summer and winter semesters. Typically, the meaning of the $20{ }^{\circ} \mathrm{C}$ in summer that the weather is cool, but the meaning for the same degree in winter that the weather is warm. Consequently, the meaning for the same data/measurements has different meanings in different phases or levels. So, this measurement can be fuzzifier to 0.2 as a value lies in $[0,1]$, the problem appears when we need to defuzzification 0.2 to the original information, we lose the full meaning of $20{ }^{\circ} \mathrm{C}$ whether in summer or winter semester. Here, the phase term overcomes the presented problem by representing the stages, levels, or phases of information in the complex fuzzy form as a value lies in the unit disk. Also, this research covered some notions that were not introduced in preceding research, such as $\mathrm{CF}$ subgruopoid, lattice of $\mathrm{CF}$ subgruopoid, CF (left, right) ideal.

As future research, we may extend this work to present several notions as complex intuitionistic fuzzy subgroups, complex $q$-fuzzy subgroups, complex fuzzy subring of a ring, and study the homomorphism and other representative properties between two algebraic structures of preceding notions. Also, we may use our assumption in several fields of mathematics to introduce complex fuzzy topology, complex fuzzy partial differential equation, and investigate its properties with advantages of using periodic nature in the complex numbers.

\section{Cnoclusion}

In this paper, we introduced some notions in the theory of fuzzy groups by adding the co-domain of membership function from $[0,1]$ to unit disk in the complex plane. The advantage of this notions lies in its ability to present several levels by using the phase term (periodicity property) in the complex number. Some notions and its properties under complex fuzzy restrictions were studied and investigated sequentially, to introduce the complex fuzzy subgroups in more details and in more general form. As a future research, we may extend our work to the suggestion theory of $Q$-complex fuzzy group, complex intuitionistic fuzzy group, neutrosophic complex fuzzy groups and subgroups. Also, this research built the basic structure of $\mathrm{CF}$ group and to present its properties. As future research, the present structure can be employed to generalize algorithms and classifications and may solve problems in the field of medical diagnosis, pattern recognition, and classification knowledge by highlighting and considering a periodic information.

\section{References:}

[1] Zadeh L.A, Fuzzy Sets, Inform. and Control., Vol.8, 1965, pp. 338-353.

[2] Osipov A., On Some Fuzzy Classification Algorithms and the AEC Model, WSEAS TRANSACTIONS on SYSTEMS, Vol.19, 2020, pp. 168-177, doi:10.37394/23202.2020.19.22.

[3] Arulmurugan R., Optimization of perturb and observe based fuzzy logic MPPT controller for independent PV solar system, WSEAS TRANSACTIONS on SYSTEMS, Vol.19, 2020, pp. 159-167, doi:10.37394/23202.2020.19.21.

[4] Nakrani N. M.,Joshi M. M., Fuzzy based Adaptive Dimension Parking Algorithm Including Obstacle Avoidance for Autonomous 
Vehicle Parking, WSEAS Transactions on Computers, Vol.19, 2020, pp. 277-284, doi:10.37394/23205.2020.19.33.

[5] Bovchaliuk S., Tymchuk S., Shendryk S., Shendryk V., The Fuzzy Control Automation Architecture of Parallel Action for the Intelligent Smart Grid Networks, WSEAS Transactions on Computers, Vol.19, 2020, pp. 21-25, doi:10.37394/23205.2020.19.3.

[6] Rosenfeld A, Fuzzy Groups, Math .Anal .Appl., Vol.35, 1971, pp. 512-517.

[7] Anthony J.M, Sherwood H, Fuzzy Groups Redefined, J. Math .Anal .Appl., Vol.69, 1979, pp. 124130.

[8] M.Wu. W, Normal Fuzzy Subgroups, Fuzzy math., Vol.1, 1981, pp. 21-30.

[9] Makherjee N.P, Bhattacharya, Fuzzy Normal Subgroups and Fuzzy Cosets, Inform. Sci., Vol.34, 1984, pp. 225-239.

[10] Murali V, Makamba B.B, Counting the Number of Fuzzy Subgroups of an Abelian Group of Or$\operatorname{der} p^{n} q^{m}$, Fuzzy Sets and Systems, Vol.144, No.3, 2004, pp. 459-470.

[11] Murali V, Makamba B.B, Fuzzy Subgroups of Finite Abelian Groups, Far East Journal of Mathematical Sciences, Vol.14, No.1, 2004, pp. 113 125.

[12] Alkouri A. U. M, Salleh A. R, Complex Atanassov's intuitionistic fuzzy sets, Proceedings of AIP Conference Proceedings 1482, 2012, pp. 464-470, doi:10.1063/1.4757515.

[13] Alkouri A. U. M, Salleh A. R, Complex Atan,assov's Intuitionistic Fuzzy Relation, $A b$ stract and Applied Analysis, Article ID 287382, 2013, pp. 1-18.

[14] Alkouri A. U. M, Salleh A. R, Linguistic variables, hedges and several distances on complex fuzzy sets, Journal of Intelligent and Fuzzy Systems, Vol.26, No.5, 2014, pp. 2527-2535.

[15] Al-Qudah Y, Hassan N, Complex multi-fuzzy soft set: Its entropy and similarity measure, IEEE Access, Vol.6, 2018, pp. 65002-65017.

[16] Al-Qudah Y, Hassan N, Operations on complex multi-fuzzy sets, Journal of Intelligent and Fuzzy Systems, Vol.33, No.3, 2017, pp. 1527-1540.

[17] Li C, Tu. C.-H, Complex neural fuzzy system and its application on multi-class prediction -
A novel approach using complex fuzzy sets, IIM and multi-swarm learning, Applied Soft Computing, Vol.84, 2019.

[18] Ramot D, Milo R, Friedman M, Kandel A, Complex fuzzy sets, IEEE Transaction on Fuzzy Systems, Vol.10, No.2, 2002, pp. 171-186.

[19] Singh P.K, Bipolar $\delta$-equal complex fuzzy concept lattice with its application, Neural Computing and Applications, Vol.32, 2020, pp. 24052422.

[20] Tamir D. E, Kandel A, Axiomatic theory of complex fuzzy logic and complex fuzzy classes, Int. J. of Computers Communications and Control, Vol.6, No.3, 2011, pp. 562-576.

[21] Tamir D. E, Last M, Kandel A, Generalized complex fuzzy propositional logic, The World Conference on Soft computing, San Francisco, 2011.

[22] Tamir D. E, Last M, Teodorescu H. N, Kandel A, Discrete complex fuzzy logic, In Proceedings of NAFIPS, North America, 2012.

[23] Tamir D. E, Lin J, Kandel A, A new interpretation of complex membership grade, International Journal of Intelligent Systems, Vol.26, 2011, pp. 285-312.

[24] Alsarahead M. O, Ahmad A. G, Complex Fuzzy Subgroups, Applied Mathematical Sciences, Vol.11, No.41, 2017, pp. 2011-2021.

[25] K. A. Dib, On fuzzy spaces and fuzzy group theory, Inform. Sci., Vol.80, 1994, pp. 253-282.

[26] J. M. Anthony and H. Sherwood, A characterization of fuzzy subgroups, Fuzzy Sets and Systems, Vol.7, 1982, pp. 297-305.

[27] I. Guessarian, A survey on classes of interpretations and some of their applications, in: M. Nivat, J.C. Reynolds, Eds., Algebraic Methods in Semantics, Cambridge University Press, London, 1985, pp. 383-409.

[28] M. Mashinchi and M. Mukaidono, Algebraic knowledge classification, J. Fuzzy Math., Vol.2, 1994, pp. 233-247.

[29] S-C Cheng and J. N. Mordeson, Applications of fuzzy algebra in automata theory and coding theory, Fifth IEEE International Conference on Fuzzy Systems, Proceedings vol.1, 1996, pp. 125129. 
[30] J. N. Mordeson, D. S. Malik and S-C Cheng, Fuzzy Mathematics in Medicine, Studies in Fuzziness and Soft Computing, Physica-Verlag, A Springer-Verlag Company, Vol.55, 2000.

[31] Zhang G, Dillon T. S, Cai K. Y, Ma J, Lu J, $\delta$ Equalities of complex fuzzy relation, 24th IEEE International Conference on Advanced Information Networking and Applications-Perth, Western Australia, 2010, pp. 1218-1224.

\section{Contribution of individual authors to the creation of a scientific article (ghostwriting policy)}

Author Contributions:

Abdulazeez Alkouri has given the idea. Also he searched in previous works and wrote the draft of the paper till section three. In addition, he wrote section six and seven, then he revised the final version of the paper.

Amani Sheimat has co-operated with A. Alkouri in the draft of the paper till section three, then she checked references details.

Murad Massa'deh has investigated the new ideas and wrote the draft of section four and five. Also he finished the first draft of the paper in Latex format. Eman A. Abuhijleh has co-operated with M. Massa'deh in the draft of section four and five, and co-operated with A. Alkouri in the final revision of the paper. Besides, she organized the team work and finally followed publishing work.

\section{Sources of funding for research presented in a scientific article or scientific article itself No fund}

\section{Creative Commons Attribution \\ License 4.0 (Attribution 4.0 \\ International, CC BY 4.0)}

This article is published under the terms of the Creative Commons Attribution License 4.0

https://creativecommons.org/licenses/by/4.0/deed.en_US 